\title{
Prediction of Concrete Strength using Floating Centroids Method
}

\author{
Lin Wang \\ Shandong Provincial Key Laboratory \\ of Network based Intelligent Computing, \\ University of Jinan, Jinan, 250022, China \\ Email:wangplanet@gmail.com
}

\author{
Bo Yang \\ ${ }^{1}$ Shandong Provincial Key Laboratory \\ of Network based Intelligent Computing, \\ University of Jinan, Jinan, 250022, China \\ 2 School of Informatics, Linyi University, \\ Linyi 276000, China \\ Corresponding Author \\ Email: yangbo@ujn.edu.cn
}

\author{
Ajith Abraham \\ ${ }^{1}$ IT4Innovations, VSB-Technical \\ University of Ostrava, Ostrava, \\ Czech Republic \\ 2 Machine Intelligence Research \\ Labs (MIR Labs), WA, USA \\ E-mail: ajith.abraham@ieee.org
}

\begin{abstract}
Concrete is viewed as the most important cementbased composite material in the field of civil engineering. Its strength is considered the most important among its mechanical properties. Although the value of strength can be directly forecasted, the estimation of strength grade remains particularly important because concrete mortar is non-uniform, and practical preparation and curing cannot be fully simulated under laboratory conditions. In this paper, concrete strength grade was predicted by using the floating centroids method neural network classifier, which removes the fixed-centroid constraint and increases the possibility of finding an optimal neural network. Experimental results show that concrete strength prediction performance is improved by employing the floating centroids method.
\end{abstract}

Index Terms-Neural Network, Floating Centroids Method, Concrete Strength

\section{INTRODUCTION}

Concrete is viewed as the most important cement-based composite material in the field of civil engineering. Concrete is a kind of artificial stone obtained by preparing, mixing, and curing a mixture of cement, aggregates, and water. Certain advantages of concrete include abundance of raw materials, low cost, and a simple production process. In addition, concrete has higher compressive strength and superior durability compared with other engineering materials. The concrete material is widely used in various applications, including civil engineering, shipbuilding, industrial machinery, ocean development, and geothermal engineering.

One of the most essential cementitious materials used in modern concrete is cement, which consists of a variety of clinker minerals and admixtures. The differences in hydration activity between different components complicate the modeling and analysis of cement hydration. Furthermore, cement hydration directly affects the development of heat release, mechanical properties, cracking resistance, and durability. Thus, predicting which properties of concrete would be affected by cement hydration remains a difficult task.

The most important among the mechanical properties of concrete is strength, which refers to the capacity of concrete to resist pressure, pull, bend, and shear. The grade of concrete is classified based on its standard compressive strength, and concrete strength is directly affected by the following: mix ratio, amount and type of cement and aggregate, and the preparation and curing process. Compressive strength is traditionally determined by preparing, curing, and testing a cubic-shaped concrete specimen. Concrete specimens are then taken out after a certain age to measure average strength. However, such a conventional experimental method consumes a large quantity of raw materials and requires a great deal of time.

The strength value of concrete and cement can be forecasted using various computational methods such as neural network [1], gene expression programming [2], support vector machines [3], and genetic programming [4]. However, the concrete mortar itself is non-uniform. Thus, practical preparation and curing cannot be fully simulated under laboratory conditions. Therefore, the estimation of strength grade, which corresponds to the range of strength, remains particularly important. Aside from direct forecasting, the prediction of concrete strength can also be transformed into a classification problem in which the concrete specimen is categorized into one of the grades. The classification task is a major research area in data mining. The supervised classification model is built from a given training set and can predict the class of the following test samples. Various classification techniques have been proposed, such as [5], [6]. In these techniques, the neural network classifier [7][10] succeeds in several practical applications and shows a great potential for application.

However, the fixed-centroid constraint decreases the likelihood of finding an optimal neural network in the traditional neural network classifier. The floating centroids method (FCM) [11]-[14] is a new neural network classifier that removes the limit of fixed-centroids by introducing several floating centroids, which are spread throughout the partition space and produced via the k-means algorithm. This method outperforms other classifiers and shows favorable application potential [11]. The high cost of the traditional experimental method for 


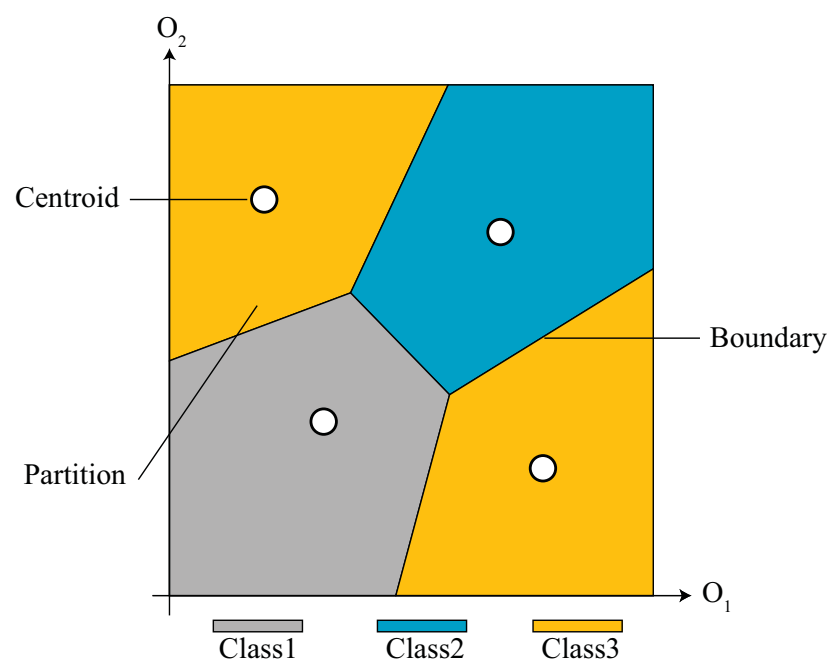

Fig. 1. A colored partition space of 3-class classification [11]. The dimension of partition space is 2 and the number of centroids is 4 .

determining concrete strength and the progress achieved by the FCM neural network classifier motivates this study to explore the process of classifying concrete strength grade using FCM.

This paper is arranged as follows. Section 2 describes the floating centroids method and its application in the prediction of concrete strength grade. Section 3 outlines and discusses our experimental results, and Section 4 concludes this paper.

\section{Methodology}

\section{A. Floating Centroids Method}

Based on the output of a neural network, the partition space is responsible for classifying the samples as a class. The classification commences when FCM generates certain floating centroids with category labels in the partition space. These floating centroids are then used to classify the mapped sample points. Partition is defined as the irregular regions around centroids. The partitions in traditional neural network classifiers are determined manually, whereas the partitions in FCM are divided automatically based on actual distribution. Therefore, natural decision boundaries exist between partitions. Certain abstract symbols of class, which are called "colors", are attached to these centroids to mark their type. The partition space that contains the colored centroids is called the "colored partition space." Fig. 1 illustrates these concepts.

A sample that needs to be classified is first mapped by the neural network to the partition space. The class of a sample is then predicted as the class of the centroid nearest to the corresponding mapped point of the sample in the partition space.

In the learning process of FCM, the task of finding the optimum neural network and minimizing the target function is performed by particle swarm optimization (PSO) [15], [16], which is a widely used and effective optimization tool inspired by the foraging of birds. PSO aims to solve global optimization problems. The "particle" represents a possible solution to a problem in the search space. In the PSO algorithm, particles fly in the search space following the best solution found by the whole population and the historical best solution found by each particle. This process is performed repeatedly until the termination condition is satisfied. The position of the best particle is an approximate optimum solution. In the FCM neural network classifier, a particle represents a vector coded from a feasible neural network. The optimization goal is to identify the best neural network and the corresponding colored partition space.

FCM performs the centroid generation process to obtain the floating centroids and color schemes. FCM covers both the calculation of centroids and the assignment of class labels. First, all training samples are mapped to the partition space by the neural network. These mapping points are called color points, which are then partitioned into a fixed number of disjoint subsets using the k-means clustering algorithm [17]. The centers of these disjoint subsets are defined as the centroids. Finally, each of these centroids is colored by one of the classes if the training points of that class comprise the majority of all points around the centroid.

The learning process of FCM attempts to find the best neural network by keeping the points in the same class as close as possible and the points in different classes as far as possible from one another [11]. Natural boundaries exist between centroids because the position and class label of centroids are computed based on actual distribution. Therefore, floating (ienvolving number and position) centroids in FCM remove the limit from the fixed centroid and increase the chance of finding the optimal neural network compared with the one-per-class SoftMax [18] and ECOC [19] methods.

\section{B. Prediction of Concrete Strength Grade}

The training data set can be obtained from the concrete experiments by setting the mix ratio of raw material and the curing age as inputs and the final strength grade as output. These training data are fed into the learning stage of FCM to obtain the optimum neural network and its corresponding colored partition space. If the strength grade of a new concrete specimen has to be categorized, its mix ratio and curing age are first mapped into the colored partition space by the constructed neural network. The category is then predicted based on the class of the nearest centroid. Fig. 2 illustrates the categorization of a sample to a certain strength grade using FCM.

\section{EXPERIMENTS}

To assess the validity of the FCM algorithm in the classification of concrete strength grade, three kinds of measurement standards are adopted in experiment: training accuracy (TA), generalization accuracy (GA), and average f-measure (Avg. FM), which are defined as follows:

$T A=\frac{\text { number of correctly classified samples in training data set }}{\text { number of total samples in training data set }}$ 


\section{The properites of concrete sample to be predicted are mapped by neural network}

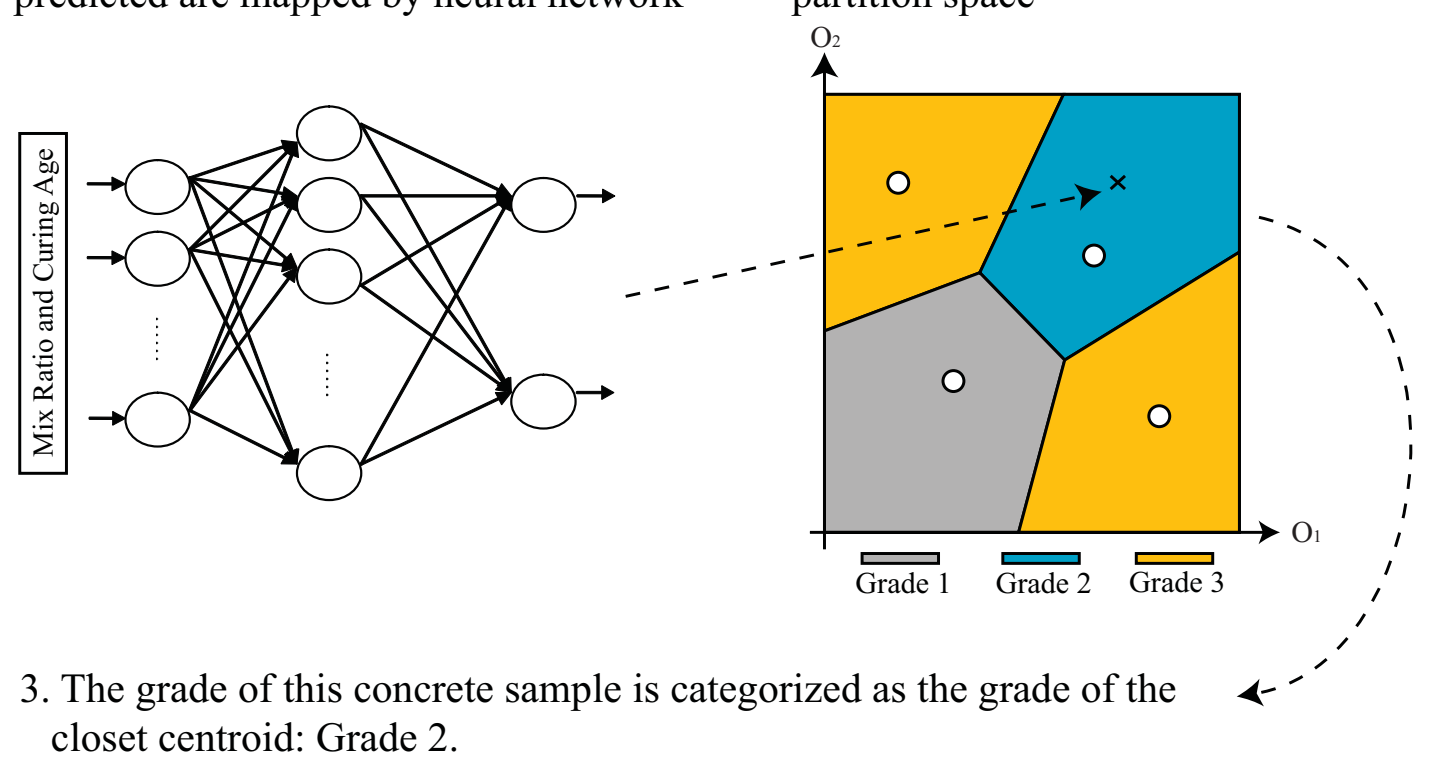

Fig. 2. Categorize a new concrete sample. The dimension of partition space is set to two and the number of classes is three.

$$
\begin{gathered}
G A=\frac{\text { number of correctly classified samples in test data set }}{\text { number of total samples in test data set }} \\
\text { Avg. } F M=\frac{\sum_{i=1}^{N}\left(2 \times \frac{\text { precision }_{i} \times \text { recall }_{i}}{\text { precission }_{i}+\text { recall }_{i}}\right)}{N}
\end{gathered}
$$

TA measures how well a method adapts the training data. A method will achieve higher TA if it adapts training data better. GA measures how well a method adapts the testing data. A method will achieve higher GA if it possesses better generalization capability. Avg. FM considers both the precision and the recall of the test to compute the score. Precision refers to the number of correct results divided by the number of all returned results, whereas recall refers to the number of correct results divided by the number of results that should have been returned. The best value for the f-measure is one, whereas the worst value is zero. Our experiments adopted Avg. FM, which calculates the mean value for all the classes.

The concrete compressive strength data set, which was selected from the UCI machine learning repository (available from the website: http://archive.ics.uci.edu/ml/), was taken as the experimental data. These data sets contained 1030 samples, and each sample consisted of eight input variables: Cement $\left(\mathrm{kg} / \mathrm{m}^{3}\right)$, Blast Furnace Slag $\left(\mathrm{kg} / \mathrm{m}^{3}\right)$, Fly Ash $\left(\mathrm{kg} / \mathrm{m}^{3}\right)$, Water $\left(\mathrm{kg} / \mathrm{m}^{3}\right)$, Superplasticizer $\left(\mathrm{kg} / \mathrm{m}^{3}\right)$, Coarse Aggregate $\left(\mathrm{kg} / \mathrm{m}^{3}\right)$, Fine Aggregate $\left(\mathrm{kg} / \mathrm{m}^{3}\right)$, and Age (day). The output variable is concrete compressive strength $(M P a)$. All samples are divided into four classes. The first class $(\mathrm{C} 1)$ consists of samples that are less than 20Mpa. The second class (C2) consists of samples that are less than $40 M p a$ but greater than 20Mpa. The third class (C3) consists of samples that are less than 60Mpa but greater than 40M pa. The fourth class (C4) consists of samples that are greater than $60 \mathrm{Mpa}$.

To balance the scale, all features are normalized using the min-max normalization method. This method performs a linear transformation that maps the original value of the feature $f$ to a new value between zero and one from the scale between the maximum value $f_{\max }$ and the minimum value $f_{\min }$ as follows:

$$
f^{\prime}=\frac{f-f_{\min }}{f_{\max }-f_{\min }}
$$

The 10-fold cross-validation is used to validate the proposed method [11]. First, each data set is split into 10 subsets randomly. Second, all samples of each class are assigned into these subsets uniformly. One subset is then selected as the testing set, whereas the remaining nine subsets are used as the entire training set. A one-time validation is conducted for this pair of training and testing sets. Finally, the procedure is repeated 10 times, and each subset is tested once. A threelayered feedforward neural network with 15 hidden neurons is then selected in the experiment. In addition, PSO is used for the optimization of the results. The following settings are adopted in real experiment: Max Generation $=10000$, Population Size $=20, \varphi 0=1, \varphi 1=1.8, \varphi 2=1.8$, VMAX $=3, m \mathrm{e}=2$, and $k=6$.

The training process of the concrete strength prediction is shown in Fig. 3, which illustrates the variation of training accuracy for all 10 validations. This figure shows that the evolution process converges gradually after 600 generations. After evolution, the optimal neural network and its corresponding colored partition spaces are obtained. The colored partition 


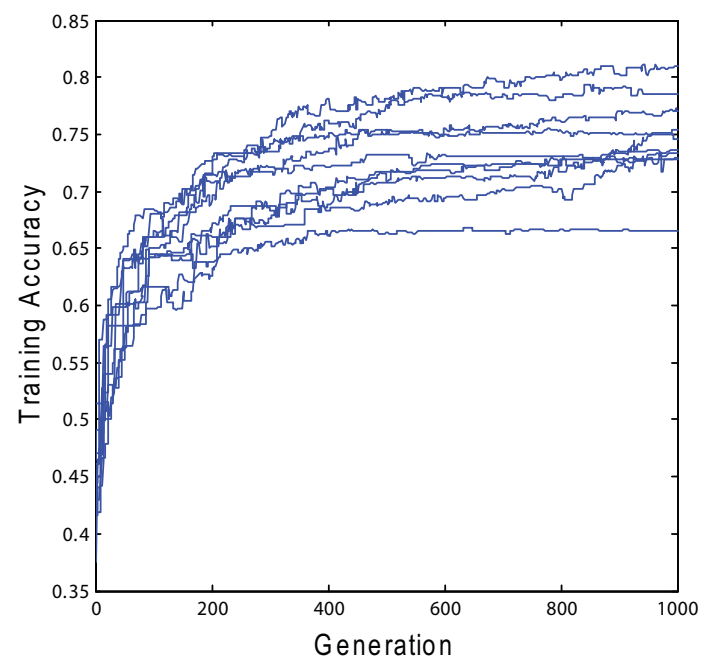

Fig. 3. The training process of concrete strength prediction on all validations.

TABLE I

RESULTS OF TRAINING ACCURACY

\begin{tabular}{ccccc}
\hline Validation & One-Per-Class & SoftMax & ECOC & FCM \\
\hline 1 & 0.304865 & 0.469189 & 0.603243 & 0.770811 \\
2 & 0.382703 & 0.608649 & 0.710270 & 0.736216 \\
3 & 0.391351 & 0.593514 & 0.608649 & 0.727568 \\
4 & 0.511351 & 0.562162 & 0.657297 & 0.754595 \\
5 & 0.655135 & 0.554595 & 0.629189 & 0.732973 \\
6 & 0.437838 & 0.609730 & 0.656216 & 0.745946 \\
7 & 0.449730 & 0.598919 & 0.574054 & 0.665946 \\
8 & 0.553514 & 0.224865 & 0.503784 & 0.785946 \\
9 & 0.095135 & 0.603243 & 0.650811 & 0.729730 \\
10 & 0.556757 & 0.441081 & 0.508108 & 0.809730 \\
\hline MEAN & 0.433838 & 0.526595 & 0.610162 & 0.745946 \\
STD & 0.156509 & 0.121530 & 0.066218 & 0.038909 \\
\hline
\end{tabular}

spaces are shown in Fig. 4, which shows that the partitions of close grades are adjacent to one another in the partition space.

FCM was compared with three other neural network classifiers: the traditional method (a simple output method for binary classification and a one-per-class method for multiclassification), SoftMax, and ECOC (exhaustive code). Table I-III depicets the accuracy results of the comparison between FCM and the other methods in terms of concrete strength prediction. The average training accuracy of FCM is evidently higher than that of the other methods in the experiments. Moreover, the smallest standard deviation is also produced by FCM, which indicates that FCM is more stable. These findings reflect that FCM fits the training data set better. The introduction of the floating centroid increases the possibility of finding good classifier during the learning process. Furthermore, FCM achieves the highest average GA and Avg. FM in the experiments, thereby indicating that FCM is fully capable of improving the capability of the neural network classifier to predict concrete strength.
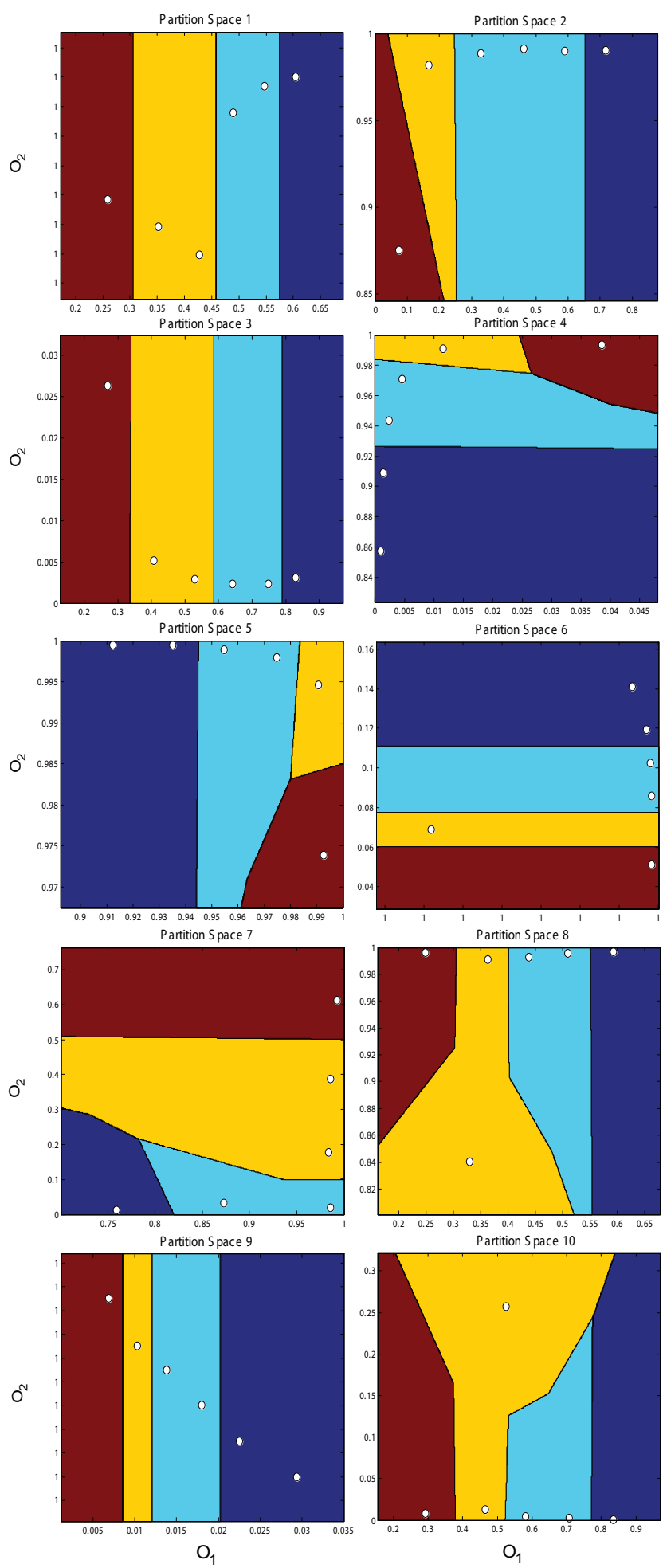

Fig. 4. The colored partition space on all validations. Each of them corresponds to a neural network. The blue partition is $\mathrm{C} 1$. The sky blue partition is $\mathrm{C} 2$. The yellow partition is $\mathrm{C} 3$ and the red partition is $\mathrm{C} 4$. 
TABLE II

RESUlts OF GENERALIZATION ACCURACY

\begin{tabular}{ccccc}
\hline Validation & One-Per-Class & SoftMax & ECOC & FCM \\
\hline 1 & 0.333333 & 0.428571 & 0.561905 & 0.542857 \\
2 & 0.438095 & 0.466667 & 0.552381 & 0.609524 \\
3 & 0.228571 & 0.638095 & 0.552381 & 0.780952 \\
4 & 0.276190 & 0.523810 & 0.561905 & 0.761905 \\
5 & 0.504762 & 0.599999 & 0.542857 & 0.780952 \\
6 & 0.514286 & 0.571429 & 0.495238 & 0.704762 \\
7 & 0.314286 & 0.609524 & 0.419048 & 0.761905 \\
8 & 0.533333 & 0.247619 & 0.447619 & 0.761905 \\
9 & 0.009524 & 0.542857 & 0.485714 & 0.790476 \\
10 & 0.361905 & 0.438095 & 0.514286 & 0.638095 \\
\hline MEAN & 0.351429 & 0.506667 & 0.513333 & 0.713333 \\
STD & 0.159897 & 0.116366 & 0.050485 & 0.086760 \\
\hline
\end{tabular}

TABLE III

Results of Average F-Measure

\begin{tabular}{ccccc}
\hline Validation & One-Per-Class & SoftMax & ECOC & FCM \\
\hline 1 & 0.227075 & 0.156250 & 0.375291 & 0.532906 \\
2 & 0.198696 & 0.260237 & 0.430754 & 0.571778 \\
3 & 0.218927 & 0.369343 & 0.38144 & 0.738408 \\
4 & 0.377857 & 0.329517 & 0.499901 & 0.805318 \\
5 & 0.414323 & 0.414309 & 0.367088 & 0.819663 \\
6 & 0.312174 & 0.329283 & 0.384093 & 0.739809 \\
7 & 0.203474 & 0.354955 & 0.325974 & 0.647011 \\
8 & 0.331278 & 0.125848 & 0.183277 & 0.698486 \\
9 & 0.005102 & 0.301441 & 0.290078 & 0.763203 \\
10 & 0.276863 & 0.152318 & 0.345441 & 0.490975 \\
\hline MEAN & 0.256577 & 0.279350 & 0.358334 & 0.680756 \\
STD & 0.115649 & 0.101514 & 0.083908 & 0.115290 \\
\hline
\end{tabular}

\section{CONCLUSION}

In this paper, we explored the process of classifying concrete strength grade using the neural network classifier with FCM, which removes the fixed-centroid constraint and increases the possibility of finding an optimal neural network. This method divides the output partition space into many irregular partitions using floating centroids. By setting the mix ratio of raw material and the curing age as inputs and the final strength grade as output, FCM translates the properties of the concrete sample to a grade of strength. The strength grade of the sample is predicted based on the grade of the centroid nearest to the mapped point.

To evaluate the performance of the proposed approach, the well-known concrete compressive strength data set is selected to compare several measurements. Experimental results manifest that prediction performance is improved by employing FCM.

\section{ACKNOWLEDGMENT}

This work is supported by National Key Technology Research and Development Program of the Ministry of Science and Technology under Grant 2012BAF12B07-3. National Natural Science Foundation of China under Grant No. 61173078, No. 61203105, No. 61173079, No. 61070130, No. 60903176. Provincial Natural Science Foundation for Outstanding Young Scholars of Shandong under Grant No. JQ200820. Shandong
Provincial Natural Science Foundation, China, under Grant No. ZR2010FM047, No. ZR2012FQ016, No. ZR2012FM010. Program for New Century Excellent Talents in University under Grant No. NCET-10-0863. This work is partially supported in the framework of the IT4 Innovations Centre of Excellence project, reg. no. CZ.1.05/1.1.00/02.0070 by operational programme 'Research and Development for Innovations' funded by the Structural Funds of the European Union and state budget of the Czech Republic, EU.

\section{REFERENCES}

[1] I-Cheng Yeh, "Modeling of strength of high performance concrete using artificial neural networks," Cement and Concrete Research, vol. 28, no. 12, pp. 1797-1808, 1998.

[2] MH Severcan, "Prediction of splitting tensile strength from the compressive strength of concrete using GEP", Neural Computing \& Applications, vol. 21, no. 8, pp. 1937-1945, 2012.

[3] Xu-chao Shi, Yi-feng Dong, "Support vector machine applied to prediction strength of cement", 2nd International Conference on Artificial Intelligence, Management Science and Electronic Commerce, pp. 15851588, 2011.

[4] Mustafa Saridemir, "Genetic programming approach for prediction of compressive strength of concretes containing rice husk ash", Construction And Building Materials, vol. 24, no. 10, pp. 1911-1919, 2010.

[5] V. N. Vapnik, The Nature of Statistical Learning Theory, Springer Verlag, 1995.

[6] J. R. Qinlan, "Introduction of decision trees", Machine Learning, vol.1, no.1, pp.86-106, 1986.

[7] Lu Hongjun, Setiono Rudy and Liu Huan, "Effect data mining using neural networks", IEEE Transaction on knowledge and data engineering, vol.8, no.6, pp.957-961, 1996.

[8] B. B. Misraa, S. Dehurib, P. K. Dashc and G. Pandad, "A reduced and comprehensible polynomial neural network for classification", Pattern Recognition Letters, vol.29, no.12, pp.1705-1712, 2008.

[9] Gao Daqi and Ji Yan, "Classification methodologies of multilayer perceptrons with sigmoid activation functions", Pattern Recognition, vol.38, no.10, pp.1469-1482, 2005.

[10] Yasser F. Hassan, "Rough sets for adapting wavelet neural networks as a new classifier system", Applied Intelligence, vol. 35, no. 2, pp. 260-268, 2011.

[11] Lin Wang, Bo Yang, Yuehui Chen, etc., "Improvement of neural network classifier using floating centroids", Knowledge and Information Systems ,vol.31, no.3, pp. 433-454, 2012.

[12] Gabriela Czibula, Istvan Gergely Czibula and Radu Dan Gaceanu, "Intelligent data structures selection using neural networks", Knowledge and Information Systems, vol.34, no.1, pp.171-192, 2011.

[13] Lei Zhang, Lin Wang, Xujiewen Wang, Keke Liu and Ajith Abraham, "Research of Neural Network Classifier Based on FCM and PSO for Breast Cancer Classification", HAIS 2012, Part I, Lecture Notes in Computer Science, vol. 7208, pp. 647-654, 2012.

[14] Ireneusz Czarnowski, Piotr Jedrzejowicz, "Agent-Based Approach to RBF Network Training with Floating Centroids", the 4th International Conference on Computational Collective Intelligence, pp. 453-462, 2012.

[15] J. Kennedy and R. C. Eberhart, "A new optimizer using paritcle swarm theory", in Proc. the Sixth Int. Symposium on Micromachine and Human Science, pp.39-43, 1995.

[16] Kan Wang, Yu Jun Zheng, "A new particle swarm optimization algorithm for fuzzy optimization of armored vehicle scheme design", Applied Intelligence, vol. 37, no. 4, pp. 520-526, 2012.

[17] J.A. Hartigan and M.A. Wong, "A k-means clustering algorithm", Applied Statistics, vol.28, no.1, pp.100-108, 1979.

[18] J. S. Bridle, "Probabilistic Interpretation of Feedforward Classification Network Outputs, with relationships to statistical pattern recognition", Neuralcomputing: Algorithms, Architectures and Applications, SpringerVerlag, pp. 227-236, 1990.

[19] TG Dietterich and G Bakiri, "Solving multiclass learning problems via error-correcting output codes", Journal of Artificial Intelligence Research, vol. 2 , no. 1 , pp. 263-286, 1995. 